\title{
Development of compact pressing system for muscle activity evaluation in force myography
}

\author{
Kazuhiro FUJISAKI*, Ayane KONDO*, Kazuhiko SASAGAWA* and Takeshi MORIWAKI* \\ *Department of Intelligent Machines and System Engineering, Hirosaki University \\ 3 Bunkyo-cho, Hirosaki, Aomori 036-8561, Japan \\ E-mail: fujiwax@hirosaki-u.ac.jp
}

Received: 11 August 2021; Revised: 21 September 2021; Accepted: 11 October 2021

\begin{abstract}
Muscle activities are usually evaluated as the subjects of motion analysis in human performance surveys. Forcemyography (FMG), which has a similar objective to electromyography, has been proposed and applied in motion analysis. Contact pressure sensors were adopted to construct the FMG device, designed for measuring muscular activities in motion. The essential factors for the detection of FMG are changes in the thickness and hardness of muscles during motion with muscle activity. This study proposed a simple structure, which is applicable as an FMG sensor, to selectively measure the hardness changes attributable to muscle activity. In addition, compact FMG sensors were manufactured by focusing on the hardness measurements by means of pressure sensing. Sensor characteristics in the dumbbell curl posture and walking motion were investigated, and some applications were provided in this study. The changes in hardness expressed the variations in muscle activity of these motions. The usability and availability of the sensor unit were confirmed in the experiments focused on muscle activity.
\end{abstract}

Keywords : Muscle activity, Contact force, Hardness, Portable device, Motion analysis

\section{Introduction}

Muscle activities are usually evaluated in motion analysis for performance surveys in the sports and rehabilitation fields. Quantification of visible information in human activities is often performed according to various measurement methods, such as motion capture systems using fixed cameras and wearable inertial and geomagnetic sensors. However, the quantification of invisible information, such as forces acting in the body with human motion, is difficult, and limited methods for estimation are adopted in actual fields. Electromyography is well known as a unique measurement technique for estimating muscle activity during human activities and is generally used for evaluating the performance of human motions in sports and in the medical and healthcare fields. Surface electromyography (sEMG) is a noninvasive and direct measurement method used to conveniently evaluate muscle activity. Recently, wearable sEMG devices have been developed and employed in a wider range of fields for motion analysis. The difficulties in sEMG applications are the amplification of imperceptible signals from active muscles and the data processing of these high-frequency patterns to reduce noise (Chowdhury et al., 2013). The contact condition between the electrodes and the body surface must be given considerable care to maintain stable electrical characteristics. However, force myography (FMG) has been proposed and applied in motion analyses (Xiao and Menon, 2019) as the same kind of objective for sEMGs, such as hand motion analyses (Li et al., 2012; Ferigo et al., 2017) and gait analyses (Jiang et al, 2018; Godiyal et al., 2019). The comparison and combination of sEMG and FMG has been studied in recent years (Connan et al., 2016; Jiang et al., 2017; Jiang et al., 2020). Contact pressure sensors were adopted to construct the FMG device, which was utilized to measure the muscular activities of hand motions. The contact pressure patterns detected from the pressure sensor array, arranged around the forearm, were altered depending on the combination of muscular work.

The essential factors for the detection of FMG are changes in the thickness and hardness of muscles during motion with muscle activity. The hardness for evaluating muscle activity is usually measured using ultrasound elastography (Brandenburg et al., 2014; Yanagisawa et al., 2011), and a piezoelectric resonance sensor is developed (Han and Kim, 2013). Magnetic resonance elastography (Heer et al., 2003; Muraki et al., 2010) has also been used to measure biological 
tissues. Muscular activity, which supports the tensile load to hold a weight, is related to the Young's modulus of the muscle tissue, especially in isometric contractions. It is possible to evaluate muscle activities focused on muscle load if the Young's modulus can be measured as changes in muscle hardness during several motions. This study proposed a simple structure that is applicable as an FMG sensor for selectively measuring the hardness change of body segments attributable to muscle activity. Compact FMG sensors were designed by focusing on hardness measurements using pressure sensing. The sensor characteristics were examined, and some applications are provided in this study.

\section{Materials and methods}

\subsection{Method}

A durometer-type FMG sensing system with light and good portability was designed and developed in this study. It was constructed from a serially concatenated thin pressure sensor and an elastic compression spring, which was placed inside a socket wall. The component-design model is shown in Fig. 1. When the sensor contacts the body surface, a reaction force is generated from the compressed tissue, including the muscles present under the skin. The reaction force is significantly dependent on the muscle hardness. If the hardness is determined under the assumption that is only considered with the Young's modulus of the muscle in the compression direction, as illustrated in Fig. 2, the reaction force $F$ is simply calculated from Eq. (1).

$$
F=k_{p}(\lambda-\delta)=k_{s} \delta
$$

where $k_{p}$ and $k_{s}$ are the values of the Young's modulus of the compression spring and subject, respectively; $\lambda$ is the initial amount of protrusion of the indenter, and $\delta$ is the indentation depth under the elastic deformation behavior. Therefore, the elastic characteristics of the subject, including the change in Young's modulus of the body in relation to muscle work, can be estimated using Eq. (2).

$$
k_{s}=k_{p} F /\left(k_{p} \lambda-F\right)
$$

In actual use, calibration tests of the pressing system constructing the spring and contact pressure sensors are required to measure $F$ from the sensor signal.

\subsection{Sensor device}

Recently, thin and flexible pressure sensors have been widely used in the field of haptic measurements (Sasagawa et al., 2017; Kasai et al., 2018). This type of pressure sensor has the characteristic of change in electrical resistance. A pressure sensor (FSR Model 400 short, Interlink Electronics) was used to miniaturize the sensor units. A trapezoidal compression spring was used to contact the sensor. The socket, including the sensor and spring, was designed and fabricated using a 3D printer (Form 3, Formlabs). Fig. 2 illustrates the sensor system. The sensor had a diameter of 20 $\mathrm{mm}$, a thickness of $13 \mathrm{~mm}$ without compression, and a weight of $3 \mathrm{~g}$ without cables. The change in electric resistance under compression conditions was detected as a voltage change by using a multiple voltage-dividing circuit with fixed resistors of $4.7 \mathrm{k} \Omega$, which was selected considering the measurement range of the pressure sensor adjusted with the experiments. The sensor output signal was connected to the analog input port on a microcomputer. Real-time data display and data processing were performed in self-produced software using a Bluetooth communication port. 


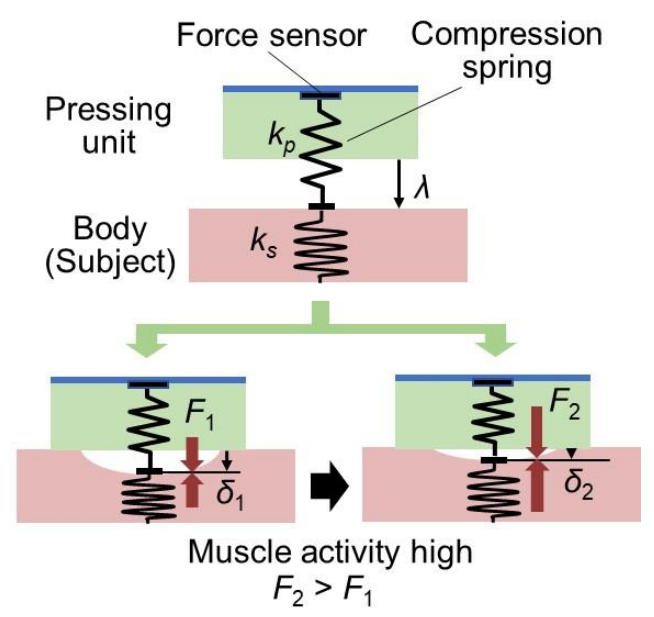

Fig.1 Mechanism of hardness measurement in the pressing system with elastic spring.
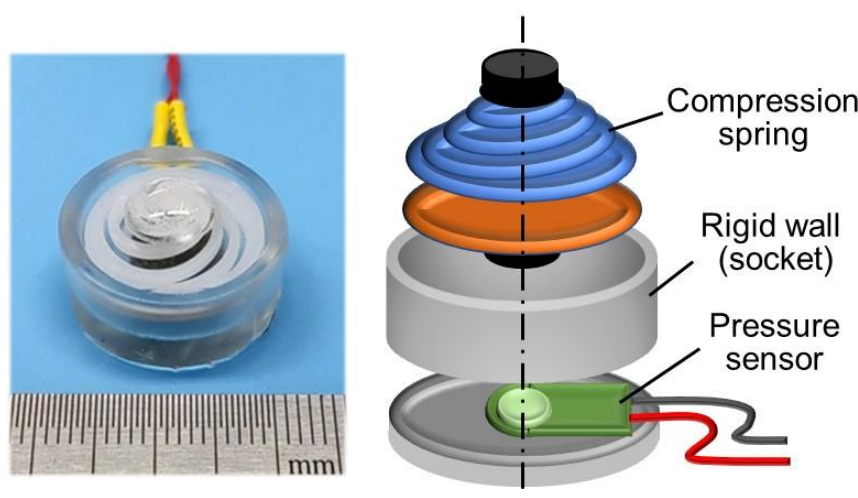

Fig.2 The structure of pressing system including main components of pressure sensor and compression spring.

\subsection{Calibration}

Compression tests were conducted to determine the force values from the sensor signals using a simple weighing equipment. The load-signal curve obtained from 0 to $15 \mathrm{~N}$ is illustrated in Fig. 3(a). The sensor signal can be directly transferred to the force according to the curve. The reaction force is determined by the force equivalent to sensor compression. Although the sensor was designed by considering the linear spring, the linear characteristics were limited; hence, a measurement error occurred in the case of the hard material. Fig. 3(b) illustrates the force-displacement curve of the sensor and several samples. The reaction forces were $1.06 \mathrm{~N}$ in the soft spring, $2.28 \mathrm{~N}$ in the compression spring; same as the sensor component, and $3.53 \mathrm{~N}$ in the silicone gel. The appropriate applicable range of the sensor was approximately $2 \mathrm{~N}$ of the reaction force. Furthermore, the values of hardness $k_{s}$ of the subject are calculated based on the linear assumption of both the sensor deformation and reaction force caused by muscle activity, according to Eq. (2). The changes in the $k_{s}$ values were investigated for several motions with muscle forces in this study.

\subsection{Experimental procedure}

Two experiments with actual measurements were conducted to investigate the sensor characteristics, focusing on the quantitative capability and stability during motion. Fig. 4 illustrates each sensor position focused on the muscle activity of the (a) biceps brachii and (b) anterior tibial muscle. The sensor was attached locally using rubbery kinesiology tape to compress the body surface while maintaining the contact condition during the experiments. All experiments were conducted as a self-experiment by the first author, who was 41 years of age at the time of the experiment.

The hardness changes induced by muscle activity were investigated in the biceps in a dumbbell curl posture, in which the elbow was held at a $90^{\circ}$ angle with changing weights, as illustrated in Fig. 4(a). The supported weight of $4 \mathrm{~kg}(39 \mathrm{~N})$, using a rope fixed at the wrist, showed changes in the moderate ascending motion, with no postural change. The supported weight was measured using a weight meter located under the weight. The measurements were performed in two instances: (1) while lifting the weight gradually by self and unloading after a short holding time, and (2) by cyclic lifting, which comprises a combination of loading and unloading, taking $10 \mathrm{~s}$ each for lifting without an obvious holding time. The sampling frequency was dependent on the weight meter at approximately $5 \mathrm{~Hz}$.

A gait analysis was conducted to confirm the portable stability. Three sensors were attached to the lower limb focused on the anterior tibial muscle activity, while walking with slippers, as illustrated in Fig. 4(b). The two sensors were thintype pressure sensors, which are the same sensors used in the pressing unit, placed on the insole to detect the timing of toe off and heel contact, respectively. The third sensor was a pressure sensor unit attached to the anterior tibial muscle. The experiment was conducted during normal walking, including on flat roads and ascending stairs. The sampling frequency was $20 \mathrm{~Hz}$, and the total recording time was $20 \mathrm{~s}$. 


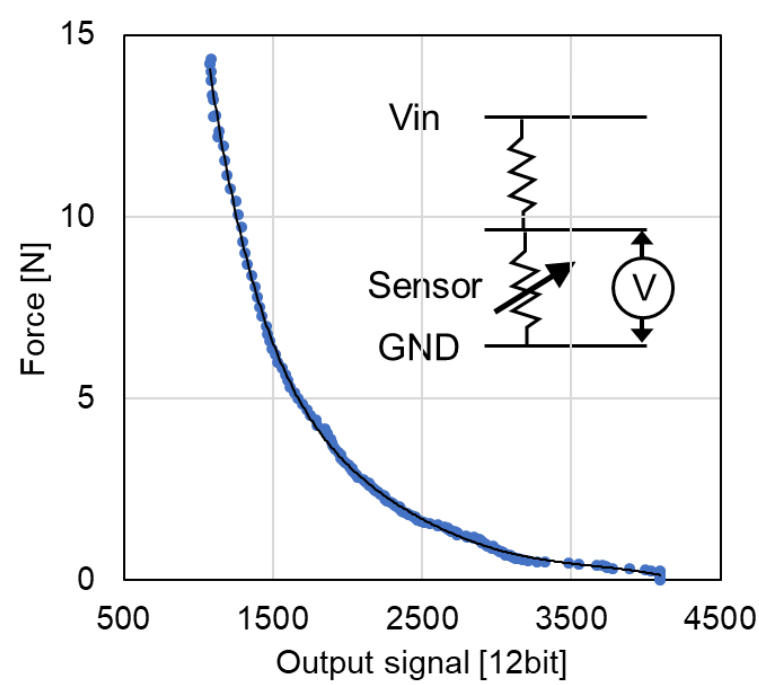

(a)

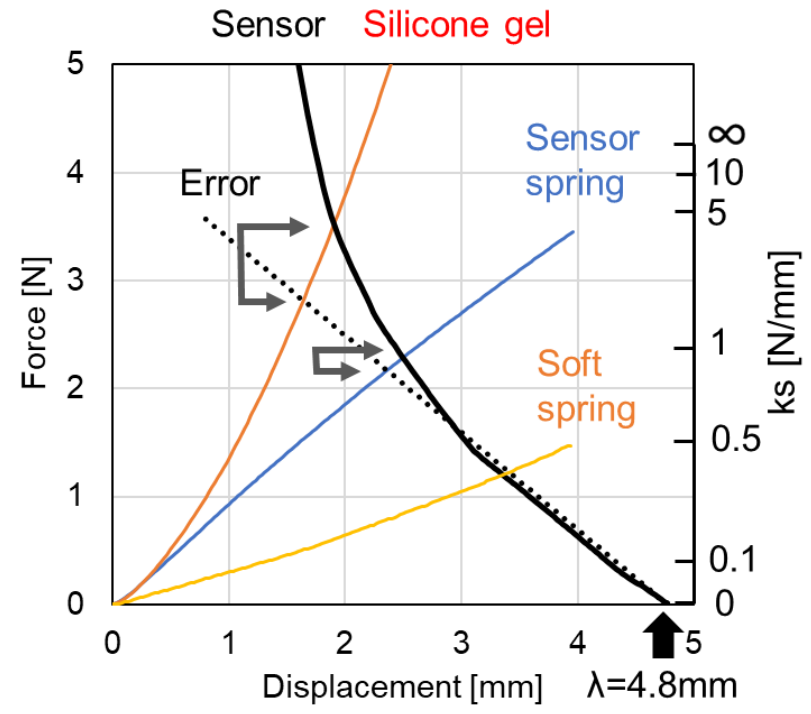

(b)

Fig. 3 Characteristics of sensor, (a) Calibration curve to determine the pressing force from sensor signal of $12 \mathrm{bit}$ A/D (04095), (b) The force-displacement curve measured in compression tests are presented in the case of three samples of silicone gel, compression spring used in the sensor, and soft spring. The reaction forces are measured as the values of intersecting points to the sensor characteristic curve, and the hardness values $k_{\mathrm{s}}$ are calculated from linear assumption Eq. (2).

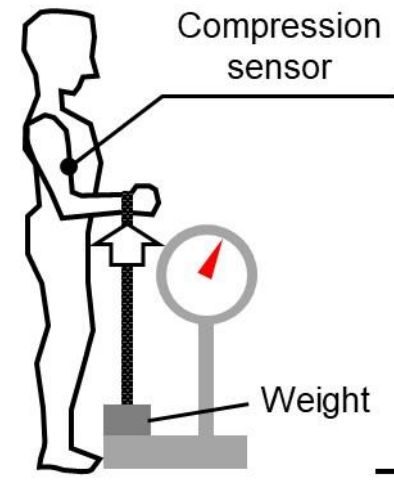

(a)

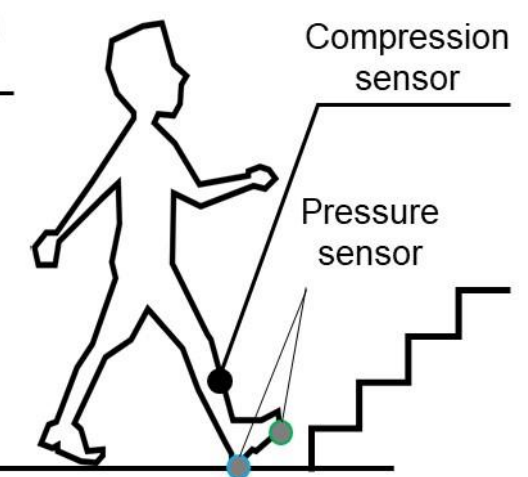

(b)

Fig. 4 Experimental posture and sensor positions for (a) biceps brachii when lifting weight and (b) anterior tibial muscle when walking on flat road and stair ascent.

\section{Result}

Fig. 5 illustrates the hardness change depending on the bicep activity during weightlifting using the dumbbell curl posture. The reaction force varied in the range of 1.29-1.43 $\mathrm{N}$ during the experiment. The change in hardness in the case of natural lifting, which took $3 \mathrm{~s}$ for loading, $2 \mathrm{~s}$ for holding, and $2 \mathrm{~s}$ for unloading, is illustrated in Fig. 5 (a). In addition, the relationship between hardness and supported weight was almost linearly related, with $\mathrm{R}^{2}=0.993$, in the loading process. The hardness changed with an increase in muscle load. The hardness slightly decreased during the holding time. In addition, the hardness decreased nonlinearly during the rapid unloading process. Fig. 5(b) illustrates the results of cyclic lifting for the three trials. The tendencies for all lifts were almost linear. The hardness values measured during the unloading process were slightly higher than those during the loading process. 
Fig. 6 illustrates the change in hardness measured for the activities of the anterior tibial muscle while walking. The reaction force varied from 1.32 to $2.32 \mathrm{~N}$. Obvious differences were observed in the hardness between the cases of walking on a flat road and while ascending stairs. The hardness drastically changed during the stance phase during flat road walking. In the case of stair ascent, although the hardness changed in almost the same range in the stance phases, it increased in the swing phases than in other periods.

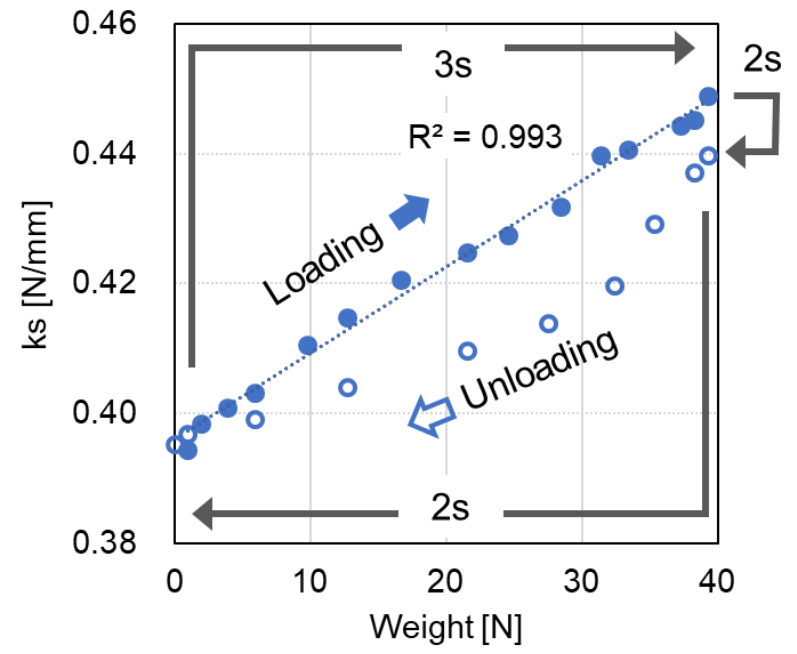

(a)

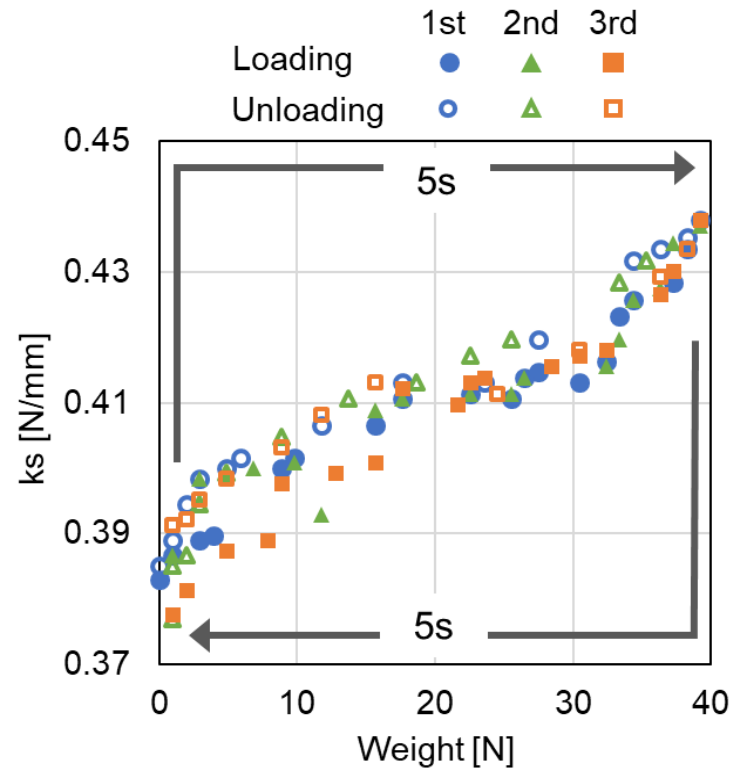

(b)

Fig. 5 Hardness change in dumbbell curl posture, (a) natural lifting of loading and unloading with holding time, (b) cyclic lifting of loading and unloading with $10 \mathrm{~s}$ frequency.

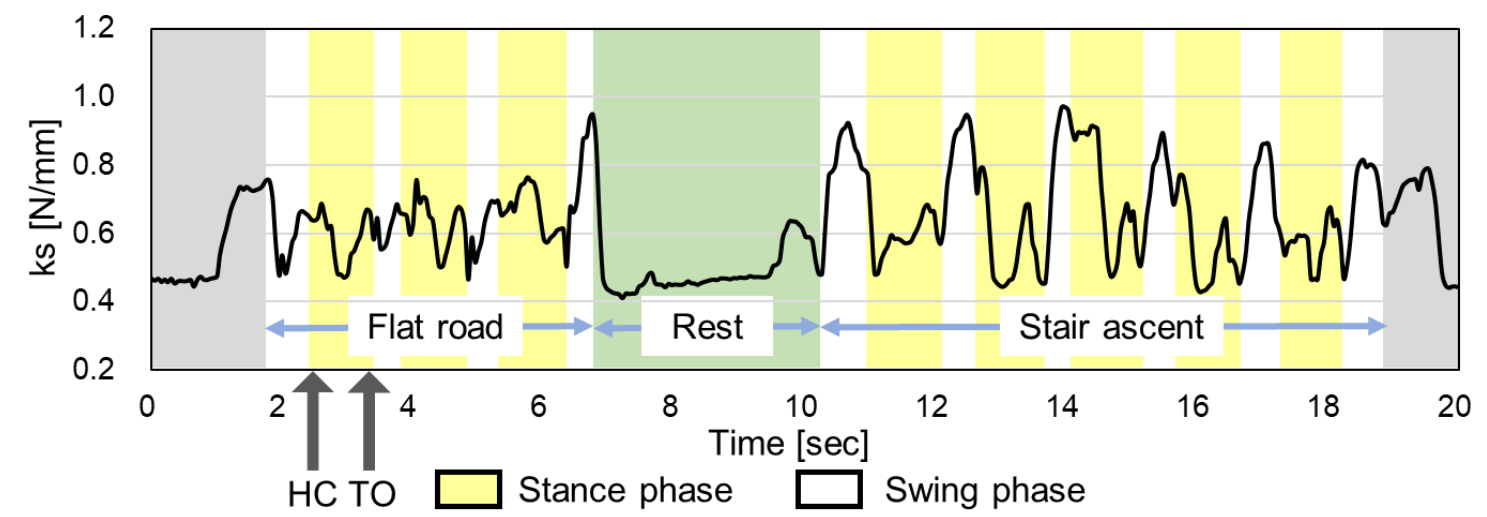

Fig. 6 Hardness change of anterior surface of leg during walking, including flat road and ascend stair.

\section{Discussion}

FMG is a useful technique to evaluate not only motion analysis but also muscle activities, especially where sEMG techniques are difficult to adopt. A pressing sensor system was developed, and its usability was confirmed in this study. The sampling speed was determined from the response of the thin pressure sensor and spring. The $20 \mathrm{~Hz}$ sampling adopted in the walking experiment was sufficient for comparison with the general video motion capture system. The unit design for improving the responses is required in analyzing rapid motion in sports, because the usual gait analysis was conducted under more than $100 \mathrm{~Hz}$ sampling. Although the sampling frequency can be easily altered in the software, the actual response of the sensor is strongly dependent on the components of the sensor system. The response of this type of 
sensor is considered more laggard than EMG (Belbasis and Fuss, 2018). The advantage of the pressing sensor is its longterm usability, rather than its fast response. This provides the possibility of using a sensor to evaluate the fatigue effects in weighted motions.

A general FMG device using a pressure sensor array was used as a wearable device; it was wrapped around the body segment by a band. Therefore, the thickness change in the muscles during motion affects other pressure sensors through a band tensile reaction. The design concept of this durometer-type FMG focuses on the hardness change at a specific position, which reduces the effect of changes in muscle thickness during motion. There is a certain level of quantity effect of the initial fixation condition on the hardness value, as illustrated in Fig. 5(a) and (b). The fixation stability of the sensor unit is a major issue when attempting to improve the quantitative measurements. Therefore, these results may need to be discussed, focusing on the increment and decrease in the hardness values, changing from the initial condition at this moment. However, the applications provided interesting results regarding the relationship between changes in hardness and lifting weight. The hardness values were higher during loading than during the unloading process in the case of natural lifting (a). However, in the case of cyclic lifting under time control (b), the values were higher during unloading than during the loading process. This indicates that redundant muscle activity is required to control the unloading process. Characteristic muscle activities were obtained while walking on ascending stairs. The anterior tibial muscle acts to raise the toe for foot lift and to prevent the slipper from dropping out under this condition. Although the hardness values at high levels have several errors because the sensor linearity decreased in the range of more than $2 \mathrm{~N}$, the plausibility of the analyses of muscle activities could be discussed as the hardness changes. However, reliability discussions about hardness detection of muscles located in deep areas are required, in comparison to other methods such as ultrasound elastography (Akagi and Kusama, 2015). Future studies should focus on characterizing the pressing system.

The deformation behavior of muscles, including soft tissue, is more complex than hardness changes when analyzing the relationship between muscle activity and sensor signals. This measurement theory is a simple consideration of the changes in Young's modulus for the reactive force direction; thus, quantitative measurements were only conducted based on linear elasticity phenomena. Introducing contact theory, such as Hertz's formula, may be effective in denoting the compression mechanism between the device and the human body surface to improve the quantitative measurements. However, the signal characteristics measured under our simple assumption indicated reasonable results in relation to the change in muscle stiffness behavior under loading. Therefore, this device and a simple method for muscle activity estimation are expected to be utilized for motion analyses in wide scenes, including outdoor fields.

\section{Conclusion}

A compact FMG sensor was developed by focusing on hardness measurements based on pressure sensing. The sensor characteristics in the dumbbell curl posture and walking motion were investigated, and some applications were provided in this study. The changes in hardness expressed variations in the muscle activity of these motions. The usability and availability of the sensor unit were confirmed through experiments that focused on muscle activity measurements.

\section{References}

Akagi, R. and Kusama, S., Comparison between neck and shoulder stiffness determined by shear wave ultrasound elastography and a muscle hardness meter, Ultrasound in Medicine and Biology, Vol.41, Issue 8 (2015), pp.22662271.

Belbasis, A. and Fuss, F. K., Muscle performance investigation with a novel smart compression and its validation against EMG, Frontiers in Physiology, Vol.9 (2018), Article 408, pp.1-13.

Brandenburg, J. E., Eby, S. F., Song, P., Zhao, H., Brault, J. S., Chen, S. and An, K. N., Ultrasound elastography: the new frontier in direct measurement of muscle stiffness, Archives of Physical Medicine and Rehabilitation, Vol.95, Issue 11 (2014), pp.2207-2219.

Chowdhury, R. H., Reaz, M. B. I., Ali, M. A. B. M., Bakar, A. A. A., Chellappan, K. and Chang, T. G., Surface electromyography signal processing and classification techniques, Sensors, Vol.13 (2013), pp.12431-12466.

Connan, M., Ramirez, E.R., Vodermayer, B. and Castellini, C., Assessment of a wearable force- and electromyography device and comparison of the related signals for myocontrol, Frontiers in Neurorobotics, Vol.10 (2016), Article 17, pp.1-13. 
Ferigo, D., Merhi, L. K., Pousett, B., Xiao, Z. G. and Menon, C., A case study of a force-myography controlled bionic hand mitigating limb position effect, Journal of Bionic Engineering, Vol.14 (2017), pp.692-705.

Godiyal, A. K., Singh, U., Anand, S. and Joshi, D., Analysis of force myography based on locomotion patterns, Measurement, Vol.140 (2019), pp.497-503.

Han, H. Y. and Kim, J., Active muscle stiffness sensor based on piezoelectric resonance for muscle contraction estimation, Sensors and Actuators A: Physical., Vol.194 (2013), pp.212-219.

Heers, G., Jenkyn, T., Dresner, M. A., Klein, M. O., Basford, J. R., Kaufman, K. R., Ehman, R. L. and An, K. N., Measurement of muscle activity with magnetic resonance elastography, Clinical Biomechanics, Vol.18, Issue 6 (2003), pp.537-542.

Jiang, S., Gao, Q., Liu, H. and Shull, P. B., A novel, co-located EMG-FMG-sensing wearable armband for hand gesture recognition, Sensors and Actuators A: Physical, Vol.301 (2020), No.111738, pp.1-5.

Jiang, X., Chu, K. H. T., Khoshnam, M. and Menon, C., A wearable gait phase detection system based on force myography techniques, Sensors, Vol.18, Issue 4 (2018), pp.1279-1291.

Jiang, X., Merhi, L. K., Xiao, Z. G. and Menon, C., Exploration of force myography and surface electromyography in hand gesture classification, Medical Engineering \& Physics, Vol.41 (2017), pp.63-73.

Kasai, R., Sasagawa, K., Fujisaki, K., Saito, S. and Koyama, H., Development of contact pressure and shear stresses sensor for touch panel, Mechanical Engineering Letters, Vol.4 (2018), No.18-00257.

Li, N., Yang, D., Jiang, L., Liu, H. and Cai, H., Combined use of FSR sensor array and SVM classifier for finger motion recognition based on pressure distribution map, Journal of Bionics Engineering, Vol.9, Issue 1 (2012), pp.39-47.

Muraki, T., Domire, Z. J., McCullough, M. B., Chen, Q. and An, K. N., Measurement of stiffness changes in immobilized muscle using magnetic resonance elastography, Clinical Biomechanics, Vol.25, Issue 5 (2010), pp.499-503.

Sasagawa, K. and Narita, J., Development of thin and flexible contact pressure sensing system for high spatial resolution measurements, Sensors and Actuators A: Physical, Vol.263 (2017), pp.610-613.

Xiao, Z. G. and Menon C., A review of force myography research and development, Sensors, Vol.19, Issue 20 (2019), 4557.

Yanagisawa, O., Niitsu, M., Kurihara T. and Fukubayashi, T., Evaluation of human muscle hardness after dynamic exercise with ultrasound real-time tissue elastography: a feasibility study, Clinical Radiology, Vol.66 (2011), pp.815-819. 